\title{
INOCULAÇÃO E COINOCULAÇÃO DE AZOSPIRILLUM E BRADYRHIZOBIUM, VIA SEMENTES E EM COBERTURA, NA QUALIDADE FISIOLÓGICA DE SEMENTES DE SOJA
}

\author{
José Henrique Bizzarri Bazzo $^{1 *}$, Jaqueline Monteiro² ${ }^{2}$ Jéssica de Lucena Marinho $^{3}$
}

\footnotetext{
${ }^{1}$ Docente, Departamento de Agronomia, Universidade Estadual de Londrina, Londrina-PR. *E-mail do autor correspondente: josebazzo@uel.br

${ }^{2}$ Engenheira Agrônoma, graduada no Centro Universitário Filadélfia, Londrina-PR.

${ }^{3}$ Doutoranda em Fitotecnia, Departamento de Agronomia, Universidade Estadual de Londrina, Londrina-PR.
}

Recebido: 08/04/2020; Aceito: 15/12/2020

RESUMO: A utilização de bactérias promotoras de crescimento de plantas no cultivo da soja tem favorecido a produtividade de grãos da oleaginosa. Contudo, as formas de aplicação e os efeitos desses microrganismos na produção de sementes de qualidade ainda são pouco estudados. Neste sentido, objetivou-se avaliar os efeitos da inoculação e da coinoculação de Azospirillum brasilense e Bradyrhizobium japonicum, aplicadas via tratamento de sementes e em cobertura, na qualidade fisiológica de sementes de soja. No campo, o experimento foi conduzido no delineamento em blocos casualizados, com 5 repetições, sendo testados cinco diferentes tratamentos: testemunha (sem inoculação e coinoculação); inoculação com $B$. japonicum aplicado via semente; aplicação de B. japonicum em cobertura; coinoculação com A. brasilense e B. japonicum aplicados via semente e; $A$. brasilense e B. japonicum aplicados em cobertura. Após a colheita, a qualidade fisiológica das sementes foi avaliada sob delineamento inteiramente casualizado, por meio dos seguintes testes: germinação, primeira contagem da germinação, envelhecimento acelerado e comprimento e massa seca da parte aérea e da raiz da plântula. Os dados obtidos foram submetidos a análise de variância, sendo as médias comparadas pelo teste de Tukey, a 5\% de probabilidade. Os tratamentos com inoculação das bactérias promotoras de crescimento produziram sementes de maior qualidade fisiológica em comparação à testemunha. A aplicação isolada de B. japonicum, via semente, resulta na produção de sementes de menor desempenho, enquanto a aplicação isolada de $B$. japonicum em cobertura, ou em conjunto com A. brasilense, aplicadas via semente ou em cobertura, favorecem a produção de sementes vigorosas.

Palavras-chave: Fitohormônios. Fixação biológica de nitrogênio. Germinação. Glycine max L. Merril. Vigor de sementes.

\section{INOCULATION AND CO-INOCULATION OF AZOSPIRILLUM AND BRADYRHIZOBIUM, BY SEEDS AND BY SIDE DRESSING, IN THE PHYSIOLOGICAL QUALITY OF SOYBEAN SEEDS}

\begin{abstract}
The use of plant growth-promoting bacteria in soybean cultivation has favored the grain yield of this oilseed. However, the forms of application and the effects of these microorganisms on the production of quality seeds are still poorly studied. In this sense, the
\end{abstract}


objective was to evaluate the effects of inoculation and co-inoculation of Azospirillum brasilense and Bradyrhizobium japonicum, applied by seed treatment and by side dressing, on the physiological quality of soybean seeds. In the field, the experiment was conducted under a randomized block design, with 5 replications, with five different treatments being tested: control (without inoculation and co-inoculation); inoculation with $B$. japonicum applied by seed; application of $B$. japonicum by side dressing; coinoculation with $A$. brasilense and $B$. japonicum applied by seed and $A$. brasilense and $B$. japonicum applied by side dressing. After harvesting, the physiological quality of the seeds was evaluated in a completely randomized design, using the following tests: germination, first germination count, accelerated aging, length and dry mass of the aerial part and seedling root. The data obtained were submitted to analysis of variance and the means were compared by the Tukey test, at 5\% probability. The treatments with inoculation of growth-promoting bacteria produced seeds of higher physiological quality compared to the control. The isolated application of B. japonicum, by seed, resulted in the production of seeds with lower performance, while the isolated application of B. japonicum by side dressing, or together with A. brasilense, applied by seed or by side dressing, favor the production of vigorous seeds.

Key words: Phytohormones. Biological nitrogen fixation. Germination. Glycine max L. Merril. Seed vigor.

\section{INTRODUÇÃO}

A soja, utilizada para diversas finalidades, é uma das espécies de maior importância econômica do mundo e, neste cenário, o Brasil se destaca como o maior produtor mundial desta oleaginosa, com 125 milhões de toneladas produzidas na última safra (2019/2020) (COMPANHIA NACIONAL DE ABASTECIMENTO - CONAB, 2020). Por este motivo, nos últimos anos, o país tem apresentado expressivo aumento na área cultivada, no rendimento e na produção de soja.

A crescente demanda do mercado interno e externo por grãos de soja estimulou a adoção de técnicas de manejo que aumentem a produtividade e reduzam os gastos e os impactos ambientais. Neste sentido, a produção e aquisição de sementes de elevada qualidade são fatores essenciais para um ótimo desempenho da cultura (MARCOS FILHO; KIKUTI 2006), visto que as sementes são responsáveis pelo estabelecimento e desenvolvimento inicial das lavouras (SPONCHIADO; SOUZA; COELHO, 2014), podendo favorecer a obtenção de um rendimento mais elevado.

É importante ressaltar que, vários fatores afetam a qualidade final da semente produzida, bem como a interação entre eles, dentre os quais destacam-se: a cultivar utilizada, a época de semeadura, as condições edafoclimáticas da região de cultivo e o manejo adotado (KOLCHINSKI; SCHUCH, 2003).

Além disso, para Sá (1994) a disponibilidade de nutrientes para as plantas também interfere na qualidade das sementes produzidas, pois afeta a composição química e a formação do embrião e dos tecidos de reservas, o que influencia diretamente o potencial fisiológico. $\mathrm{O}$ autor cita, ainda, que plantas bem nutridas apresentam melhores condições de 
produzir maior quantidade de sementes, e de melhor qualidade. Dentre os nutrientes, o nitrogênio $(\mathrm{N})$ se destaca na produção de sementes de qualidade, pois é constituinte estrutural de diversas biomoléculas e participa de reações metabólicas fundamentais para o crescimento e desenvolvimento vegetal (KOLCHINSKI; SCHUCH, 2004).

A absorção de $\mathrm{N}$, nutriente exigido em maior quantidade pela soja (SANTOS NETO et $a l .$, 2013), pode ser favorecida pela adição de inoculantes, via semente ou em cobertura, que possuem bactérias promotoras de crescimento, como as dos gêneros Azospirillum e Bradyrhizobium, que são capazes de colonizar superfícies radiculares, bem como tecidos internos da planta, estimulando o crescimento vegetal por meio de vários processos, incluindo a fixação biológica do nitrogênio (FBN) e a síntese de hormônios de vegetais (HUNGRIA; NOGUEIRA; ARAUJO, 2013).

A inoculação da soja com bactérias do gênero Bradyrhizobium, responsáveis pela formação de nódulos nas raízes, onde promovem a FBN por meio de interações simbióticas, é considerada uma prática sustentável. Esta dispensa a adubação nitrogenada e proporciona maior economia ao produtor rural (DALOLIO et al., 2018). É importante ressaltar que este gênero de bactérias também apresenta outras habilidades, como a promoção do crescimento das plantas, principalmente das raízes, além da FBN. As bactérias do gênero Azospirillum se destacam pelos efeitos benéficos proporcionados às plantas devido à sua capacidade de produzir fitohormônios em quantidades expressivas, o que melhora o crescimento radicular e, consequentemente, a absorção de água e nutrientes, resultando em melhor crescimento e desenvolvimento vegetal (BRZEZINSKI et al., 2014; MASCIARELLI et al., 2013).

Além da inoculação isolada, é possível realizar a coinoculação, que consiste na utilização de diferentes combinações de microrganismos (BÁRBARO et al., 2009), entre Azospirillum e Bradyrhizobium, o que pode favorecer a formação de sementes de elevada qualidade fisiológica, pois os efeitos positivos promovidos por cada gênero de bactéria são somados, podendo ser até mesmo potencializados. Benintende et al. (2010) e Dalolio et al. (2018) relatam que o uso combinado de Azospirillum e Bradyrhizobium em soja, resultou em maior crescimento, acumulação de $\mathrm{N}$ e produtividade.

A forma de inoculação e de coinoculação pode influenciar a eficiência da simbiose, visto que a aplicação via semente nem sempre é eficiente e pode ser afetada pela umidade e temperatura do solo no momento da semeadura, pelo uso de produtos químicos no tratamento das sementes que podem causar toxidez à bactéria, entre outros fatores (VIEIRA NETO et al., 2008). Neste contexto, outras formas de aplicação, como em cobertura, podem ser mais eficientes e garantir o sucesso da inoculação e da coinoculação (ZAGO et al., 2018). No entanto, trabalhos que visam relacionar os efeitos e as formas de inoculação e coinoculação de soja com Azospirillum e Bradyrhizobium e a produção de sementes de qualidade são escassos. Neste sentido, objetivou-se avaliar os efeitos da inoculação e da coinoculação de A. brasilense e B. japonicum, aplicados via tratamento de sementes e em cobertura, na qualidade fisiológica de sementes de soja. 


\section{MATERIAL E MÉTODOS}

O experimento de campo foi conduzido com a cultivar de soja 63I64RSF IPRO, em Astorga-PR, na propriedade Ouro Verde, localizada a $23^{\circ} 18^{\prime} 48^{\prime}$ ' S e 51 $41^{\prime} 21^{\prime \prime}$ O, com altitude de 656 m. O clima da região, segundo a classificação de Koppen, é do tipo Cfa, descrito como subtropical úmido com verões quentes, apresentando geadas pouco frequentes e tendência de concentração das chuvas nos meses de verão, contudo sem estação seca definida.

As características químicas do solo nas profundidades de 0,0-0,20 m, determinadas antes da instalação do experimento, estão descritas à seguir: $\mathrm{pH}=5,20 ;$ M.O. $=33,75 \mathrm{~g} \mathrm{dm}^{-3}$; $\mathrm{C}=19,58 \mathrm{~g} \mathrm{dm}^{-3} ; \mathrm{P}=11,61 \mathrm{mg} \mathrm{dm}{ }^{-3} ; \mathrm{K}^{+}=0,37 \mathrm{cmol}_{\mathrm{c}} \mathrm{dm}^{-3} ; \mathrm{Ca}^{+2}=6,08 \mathrm{cmol}_{\mathrm{c}} \mathrm{dm}^{-3} ; \mathrm{Mg}^{+2}=$ $1,62 \mathrm{cmol}_{\mathrm{c}} \mathrm{dm}^{-3} ; \mathrm{H}^{+}=7,00 \mathrm{cmol}_{\mathrm{c}} \mathrm{dm}^{-3} ; \mathrm{Al}^{+3}=0,20 \mathrm{cmol}_{\mathrm{c}} \mathrm{dm}^{-3} ; \mathrm{CTC}=15,28 ; \mathrm{V} \%=52,88 ; \mathrm{S}$ $=2,13 \mathrm{mg} \mathrm{dm}^{-3} ; \mathrm{Cu}=21,68 \mathrm{mg} \mathrm{dm}^{-3} ; \mathrm{Zn}=10,14 \mathrm{mg} \mathrm{dm}^{-3} ; \mathrm{Fe}=36,95 \mathrm{mg} \mathrm{dm}^{-3} ; \mathrm{Mn}=121,60$ $\mathrm{mg} \mathrm{dm}{ }^{-3} ; \mathrm{Na}=1,56 \mathrm{mg} \mathrm{dm}^{-3} ; \mathrm{B}=0,39 \mathrm{mg} \mathrm{dm}^{-3}$. Com base nas características químicas do solo da área experimental, foi realizada uma adubação mineral com $124 \mathrm{~kg} \mathrm{ha}^{-1}$ de $\mathrm{KCl}$ antes da semeadura, e $165 \mathrm{~kg} \mathrm{ha}^{-1}$ do fertilizante formulado 00-46-00 na semeadura.

O delineamento foi em blocos casualizados, com 5 repetições. Foram testados cinco diferentes tratamentos, sendo eles: testemunha (Test) (sem aplicação de $A$. brasilense e $B$. japonicum); B. japonicum aplicado em cobertura no estádio V4 (BC); A. brasilense e $B$. japonicum aplicados em cobertura no estádio V4 (ABC); B. japonicum inoculado na semente (BS) e A. brasilense e B. japonicum inoculados na semente (ABS).

As doses utilizadas nos tratamentos foram: inoculante B. japonicum SEMIA 5079 e SEMIA 5080 (Rizo Liq Top): $2 \mathrm{~mL} \mathrm{~kg}^{-1}$ de semente; inoculante A. brasilense (Masterfix L): 5 $\mathrm{mL} \mathrm{kg}^{-1}$ de semente. As inoculações foram realizadas no mesmo dia da semeadura. No estádio vegetativo V4 os tratamentos com A. brasilense e B. japonicum em cobertura foram aplicados com pulverizador manual, na mesma dose que foi utilizada para a inoculação da semente, em um volume de calda de $200 \mathrm{~L} \mathrm{ha}^{-1}$.

A semeadura foi realizada sob sistema semeadura direta, em área anteriormente ocupada com a cultura do milho, com espaçamento de 0,45 m entre linhas, profundidade de $4 \mathrm{~cm} \mathrm{e}$ densidade de semeadura de 15 sementes por metro linear. As parcelas foram formadas por oito linhas de $5 \mathrm{~m}$ de comprimento. Para as avaliações, foi considerada uma área útil de 10,8 $\mathrm{m}^{2}$. Os tratamentos fitossanitários para o controle de doenças e os demais tratos culturais foram realizados conforme a necessidade e recomendações para a cultura.

A colheita foi realizada 132 dias após a semeadura, quando as sementes atingiram a maturidade de colheita. As avaliações referentes a qualidade fisiológica de sementes foram conduzidas no Laboratório de Análise de Sementes do Centro Universitário Filadélfia (UniFil) - Campus Palhano, Londrina - PR, sob delineamento experimental de blocos inteiramente casualizados, com quatro repetições. Para determinação da qualidade fisiológica das sementes foram realizadas as seguintes avaliações:

Germinação: realizado com oito repetições de 50 sementes, semeadas em papel germitest ${ }^{\circledR}$ umedecido com água destilada na proporção de 2,5 vezes a massa do substrato 
seco. Os rolos de papel foram mantidos em germinador sob temperatura de $25{ }^{\circ} \mathrm{C}$. Posteriormente, foi realizada a contagem do número de plântulas normais aos cinco (primeira contagem) e aos oito dias (contagem final) após a semeadura, sendo os resultados expressos em porcentagem (BRASIL, 2009).

Envelhecimento acelerado: utilizou-se caixas de plástico $(11 \mathrm{~cm} \times 11 \mathrm{~cm} \times 3 \mathrm{~cm})$ dentro das quais foram adicionados $40 \mathrm{~mL}$ de água destilada para a obtenção de 100\% U.R. Em cada caixa foi colocada uma tela e sobre esta foram alocadas as sementes de cada repetição, em camada única e uniforme, sendo utilizadas quatro repetições por tratamento. Em seguida, as caixas plásticas foram levadas para a câmara de envelhecimento, regulada na temperatura de $41^{\circ} \mathrm{C}$, onde permaneceram por 48 horas (MARCOS FILHO; NOVEMBRE; CHAMMA, 2000). Decorrido esse período, foi instalado o teste de germinação sob temperatura de $25^{\circ} \mathrm{C}$. A contagem do número de plântulas normais foi realizada após cinco dias da semeadura (BRASIL, 2009).

Comprimento de plântula: efetuado utilizando-se quatro repetições de 20 sementes para cada tratamento. Os papéis de germinação foram umedecidos previamente com água destilada na proporção de 2,5 vezes a massa seca do papel seca. As sementes de soja foram posicionadas no terço superior do papel no sentido longitudinal. Os rolos foram acondicionados em sacos plásticos posicionados verticalmente no germinador por cinco dias a $25^{\circ} \mathrm{C}$. Ao final deste período, foi avaliado o comprimento das plântulas normais, separadas em parte aérea e raiz, com auxílio de uma régua milimetrada, sendo os resultados expressos em centímetros por plântula (NAKAGAWA, 1999).

Massa seca de plântula: foram avaliadas as plântulas normais, obtidas a partir do teste de comprimento, separando a parte aérea da parte radicular. As repetições foram acondicionadas em sacos de papel, identificados, e levados à estufa com circulação de ar forçado, e mantidas à temperatura de $80^{\circ} \mathrm{C}$ até atingirem massa constante. Após este período, cada repetição teve a massa avaliada em balança com precisão de 0,001 g, e os resultados médios foram expressos em miligramas por plântula (NAKAGAWA, 1999).

Os dados obtidos foram submetidos às análises de normalidade e homogeneidade dos erros e, posteriormente, à análise de variância. As médias dos tratamentos foram comparadas pelo teste de Tukey, a $5 \%$ de probabilidade.

\section{RESULTADOS E DISCUSSÃO}

Houve efeito das diferentes formas de aplicação de A. brasilense e B. japonicum para todas as características avaliadas. Para a primeira contagem da germinação, o tratamento com B. japonicum aplicado em cobertura apresentou o maior número de sementes germinadas em relação a inoculação deste microrganismo na semente. Os tratamentos com coinoculação de A. brasilense e B. japonicum, aplicados na semente ou em cobertura, não diferiram estatisticamente dos tratamentos com B. japonicum isolado. A testemunha apresentou o menor valor para esta variável em comparação aos demais tratamentos (Tabela 1), indicando que a ausência destas bactérias resultou na produção de sementes com menor velocidade de germinação. 
Tabela 1. Valores médios da primeira contagem da germinação (PC), germinação (GE), envelhecimento acelerado (EA), comprimento de parte aérea (CPA), massa seca de parte aérea (MSPA), comprimento de raiz (CR) e massa seca de raiz (MSR) da cultivar de soja 63I64RSF IPRO, em função dos tratamentos com Azospirillum brasilense e Bradyrhizobium japonicum. Mean values of the first germination count $(P C)$, germination $(G E)$, accelerated aging (EA), shoot length (CPA), shoot dry mass (MSPA), root length (CR) and root dry mass (MSR) of the soybean cultivar 63I64RSF IPRO, in function of the treatments with Azospirillum brasilense and Bradyrhizobium japonicum.

\begin{tabular}{cccccccc}
\hline Tratamentos & $\begin{array}{c}\text { PC } \\
(\boldsymbol{\%})\end{array}$ & $\begin{array}{c}\text { GE } \\
(\boldsymbol{\%})\end{array}$ & $\begin{array}{c}\text { EA } \\
(\boldsymbol{\%})\end{array}$ & $\begin{array}{c}\text { CPA } \\
(\mathbf{c m})\end{array}$ & $\begin{array}{c}\text { MSPA } \\
(\mathbf{m g})\end{array}$ & $\begin{array}{c}\text { CR } \\
(\mathbf{c m})\end{array}$ & $\begin{array}{c}\text { MSR } \\
(\mathbf{m g})\end{array}$ \\
\hline Test & $89 \mathrm{c}$ & $89 \mathrm{~b}$ & $78 \mathrm{~b}$ & $6,58 \mathrm{a}$ & $2,71 \mathrm{a}$ & $5,26 \mathrm{~b}$ & $0,08 \mathrm{c}$ \\
BC & $99 \mathrm{a}$ & $96 \mathrm{a}$ & $88 \mathrm{a}$ & $6,75 \mathrm{a}$ & $2,83 \mathrm{a}$ & $7,21 \mathrm{a}$ & $0,11 \mathrm{ab}$ \\
$\mathrm{ABC}$ & $97 \mathrm{ab}$ & $96 \mathrm{a}$ & $86 \mathrm{a}$ & $6,58 \mathrm{a}$ & $2,94 \mathrm{a}$ & $7,33 \mathrm{a}$ & $0,10 \mathrm{abc}$ \\
BS & $93 \mathrm{~b}$ & $92 \mathrm{ab}$ & $90 \mathrm{a}$ & $4,23 \mathrm{~b}$ & $2,35 \mathrm{~b}$ & $7,20 \mathrm{a}$ & $0,09 \mathrm{bc}$ \\
ABS & $98 \mathrm{ab}$ & $95 \mathrm{ab}$ & $90 \mathrm{a}$ & $6,63 \mathrm{a}$ & $2,83 \mathrm{a}$ & $7,68 \mathrm{a}$ & $0.12 \mathrm{a}$ \\
\hline Quadrado Médio & $67,45^{*}$ & $29,82^{*}$ & $103,42^{*}$ & $4,66^{*}$ & $0,20^{*}$ & $3,64^{*}$ & $0,01^{*}$ \\
\hline CV $(\%)$ & 2,43 & 2,84 & 2,79 & 10,82 & 5,07 & 8,41 & 11,02 \\
\hline
\end{tabular}

Nota: CV: coeficiente de variação. Médias seguidas da mesma letra na coluna não diferem entre si pelo teste de Tukey $(\mathrm{P}<0.05)$. Test $=$ testemunha; $\mathrm{BC}=B$. japonicum $\mathrm{em}$ cobertura; $\mathrm{ABC}=A$. brasilense $+B$. japonicum $\mathrm{em}$ cobertura; $\mathrm{BS}=B$. japonicum via semente; $\mathrm{ABS}=A$. brasilense $+B$. japonicum via semente. $C V$ : coefficient of variation. Means followed by the same letter in the column do not differ by Tukey's test $(P<0.05)$. Test $=$ control; $B C=B$. japonicum by side dressing; $A B C=A$. brasilense $+B$. japonicum by side dressing; $B S=B$. japonicum by seed; $A B S=A$. brasilense $+B$. japonicum by seed .

Fonte: Autoria própria. Own authorship.

Para a germinação, todos os tratamentos apresentaram valores superiores a $80 \%$, conforme o padrão estabelecido pela Instrução Normativa $N^{o} 45$ (BRASIL, 2013). No entanto, as plantas que receberam a aplicação de $B$. japonicum e $A$. brasilense $+B$. japonicum em cobertura, produziram sementes com maior porcentagem de germinação em relação a testemunha. Já os tratamentos com B. japonicum e A. brasilense $+B$. japonicum na semente, não diferiram dos tratamentos mencionados.

Para o envelhecimento acelerado, os tratamentos com aplicação de $A$. brasilense e $B$. japonicum, via semente ou em cobertura, resultaram na produção de sementes mais vigorosas em comparação à testemunha. Para as variáveis comprimento e massa seca de parte aérea, o B. japonicum aplicado individualmente via semente, quando comparado aos demais tratamentos, gerou os menores valores para as características resultando em sementes menos vigorosas.

Para o comprimento das raízes, os tratamentos que utilizaram as bactérias apresentaram valores maiores do que a testemunha, evidenciando que a aplicação desses microrganismos promotores de crescimento favorece a produção de sementes de qualidade. Para a massa seca de raiz os maiores e os menores valores foram verificados no tratamento em que a aplicação de $A$. brasilense com $B$. japonicum foi realizada via semente e no tratamento testemunha, respectivamente. 
De forma geral, a utilização de bactérias promotoras de crescimento de plantas no cultivo da soja favoreceu a produção de sementes de maior qualidade fisiológica em comparação à testemunha. Isto porque, a aplicação do B. japonicum isolado ou em conjunto com o A. brasilense, além de favorecer a absorção de $\mathrm{N}$ por meio do processo de $\mathrm{FBN}$, também estimula a produção de hormônios vegetais que beneficiam e favorecem o crescimento das plantas (DALOLIO et al., 2018).

Diversos estudos têm comprovado a eficiência das bactérias do gênero Azospirillum em produzir fitohormônios, como auxinas, giberelinas e citocininas, ou estimular a produção endógena desses fitohormônios (BOTTINI et al., 1989; MASCIARELLI et al., 2013), o que promove o alongamento das raízes, proporcionando maior área útil do sistema radicular para absorção de água e nutrientes, aumentando a resistência a seca e tornando as plantas mais vigorosas e produtivas (BRZEZINSKI et al., 2014), o que também favorece a produção de sementes com melhor desempenho fisiológico.

Dentre os tratamentos que incluíam a aplicação de $B$. japonicum isolado ou em combinação com $A$. brasilense em cobertura ou na semente, o tratamento com $B$. japonicum inoculado individualmente na semente foi o que resultou na produção de sementes de menor desempenho. Isso pode estar associado a baixa eficiência da inoculação isolada desta bactéria via semente, o que resultou na baixa nodulação. Este resultado pode ser decorrente da baixa umidade do solo no momento da semeadura e da toxidez causada pelo tratamento de sementes com fungicidas, inseticidas e micronutrientes (VIEIRA NETO et al., 2008; ZAGO et al., 2018).

Além disso, a maior disponibilidade inicial de $\mathrm{N}$ no solo, em virtude da decomposição da matéria orgânica remanescente dos cultivos anteriores, pode ter reduzido a nodulação e, consequentemente, a eficiência da FBN (HUNGRIA et al., 2006). O suprimento reduzido de $\mathrm{N}$ para as plantas, principalmente nas fases mais avançadas, pode ter resultado na produção de sementes com menor desempenho fisiológico.

Contudo, a aplicação em cobertura de B. japonicum resultou na produção de sementes de melhor qualidade, uma vez que, provavelmente, houve melhor nodulação, o que pode estar associado à melhoria das condições climáticas (maior umidade) em V4 (estádio em que foi realizada a aplicação em cobertura), ao menor efeito tóxico provocado pela forma de aplicação dos microrganismos, pois o tratamento aplicado nas sementes não entrou em contato direto com as bactérias e, também, à menor quantidade de $\mathrm{N}$ disponível no momento da aplicação, que foi feita alguns dias após a semeadura.

É importante ressaltar que, apesar da aplicação isolada de B. japonicum via semente ter apresentado resultados inferiores, a aplicação conjunta de A. brasilense e B. japonicum via semente, resultou na produção de sementes de alta qualidade, assim como os outros tratamentos, aplicados em cobertura, indicando sinergismo entre as bactérias, o que favorece o estabelecimento das mesmas. Hungria, Nogueira e Araujo (2013) ressaltam que as bactérias do gênero Azospirillum estimulam a produção de pelos radiculares, o que pode resultar na produção de uma maior quantidade nódulos nas raízes das plantas leguminosas. Como a formação de nódulos é resultante da simbiose entre B. japonicum e a planta, que inicia nos 
pelos radiculares, isto explica o melhor resultado obtido para a coinoculação em relação a inoculação isolada de B. japonicum, aplicados via semente.

Bárbaro et al. (2009) observaram em seu trabalho que, plantas de soja inoculadas com A. brasilense e $B$. japonicum, via semente, apresentaram sistemas radiculares mais desenvolvidos. De forma semelhante, Kumar e Chandra (2008), constataram o aumento do sistema radicular e, consequentemente, da nodulação em plantas de lentilha como resultado da coinoculação entre bactérias do gênero Rhizobium e Azospirillum, corroborando Hungria, Nogueira e Araujo (2013).

$\mathrm{O}$ crescimento radicular favorece a nodulação e, consequentemente, aumenta a disponibilidade e a absorção de água e nutrientes pela planta, principalmente o N, o que afeta a formação e composição química das sementes assim como seu metabolismo e vigor (CARVALHO; NAKAGAWA, 2012). Plantas bem nutridas e vigorosas, produzem sementes de maior qualidade fisiológica (MATTUELLA et al., 2018), o que explica os resultados obtidos.

Com base nos resultados, é possível afirmar que, embora os tratamentos que envolvam a inoculação e coinoculação de $A$. brasilense e $B$. japonicum em cobertura tenham resultado na produção de sementes de elevado desempenho fisiológico, a coinoculação aplicada via semente se destaca como uma alternativa viável e de melhor custo benefício para o produtor. Isto porque além de apresentar resultados satisfatórios, semelhante aos outros tratamentos citados, que resultaram na produção de sementes de elevada qualidade, este tratamento também representa o menor custo de produção. A coinoculação é feita juntamente com a semeadura, eliminando a necessidade de uma operação posterior, que seria a aplicação dos inoculantes em cobertura, que constitui um gasto a mais.

Além disso, a inoculação conjunta destes dois gêneros de bactérias minimiza os efeitos das adversidades edafoclimáticas que podem comprometer o crescimento e desenvolvimento das bactérias, garantindo assim maior eficiência da nodulação, maior crescimento vegetal e melhor qualidade das sementes produzidas.

\section{CONCLUSÃO}

A aplicação, via semente ou em cobertura, de bactérias do gênero Bradyrhizobium, isoladas ou em conjunto com Azospirillum, em soja, favorece a produção de sementes vigorosas.

A aplicação de combinada de A. brasilense e B. japonicum em soja, supera a inoculação isolada com B. japonicum via semente, resultando na produção de sementes de melhor vigor.

\section{REFERÊNCIAS BIBLIOGRÁFICAS}

BÁRBARO, I. M.; MACHADO, P. C.; BÁRBARO JUNIOR, L. S.; TICELLI, M.; MIGUEL, F. B.; SILVA, J. A. A. Produtividade da soja em resposta à inoculação padrão e coinoculação. Colloquium Agrariae, Presidente Prudente, v. 5, n. 1, p.1-7, 2009. Disponível em: http://dx.doi.org/10.5747/ca.2009.v05.n1.a0040. Acesso em: 08 abr. 2020. 
BENINTENDE, S.; UHRICH, W.; HERRERA, M.; GANGGE, F.; STERREN, M.; BENINTENDE, M. Comparación entre coinoculación com Bradyrhizobium japonicum y Azospirillum brasilense e inoculación simple con Bradyrhizobium japonicum en la nodulación, crecimiento y acumulación de $\mathrm{N}$ en el cultivo de soja. Agriscientia, Córdoba, v. 27, n. 2, p.71-77, 2010.

BOTTINI, R.; FULCHIERI, M.; PEARCE, D.; PHARIS, R. P. Identification of gibberellins A1, A3 and iso-A3 in cultures of Azospirillum lipoferum. Plant Physiology, Rockville, v. 90, n. 1, p.45-47, 1989. Disponível em: http://dx.doi.org/10.1104/pp.90.1.45. Acesso em: 04 abr. 2020 .

BRASIL. Ministério da Agricultura, Pecuária e Abastecimento. Secretaria de Defesa Agropecuária. Regras para análise de sementes. Brasília, DF: MAPA/ACS, 2009. 399 p. Disponível

em: http://www.agricultura.gov.br/arq_editor/file/2946_regras_analise_sementes.pdf. Acesso em: 04 abr. 2020.

BRASIL. Ministério da Agricultura, Pecuária e Abastecimento. Instrução Normativa $\mathbf{N}^{\circ} 45$ de 2013. Brasília, DF: MAPA/DAS/CSM, 2013. 38 p.

BRZEZINSKI, C. R.; ZUCARELI, C.; HENNING, F. A.; ABATI, J.; PRANDO, A. M.; HENNING, A. A. Nitrogênio e inoculação com Azospirillum na qualidade fisiológica e sanitária de sementes de trigo. Revista de Ciências Agrárias - Amazonian Journal of Agricultural and Environmental Sciences, Belém, v. 57, n. 3, p.257-265, 2014. Disponível em: http://dx.doi.org/10.4322/rca.ao1391. Acesso em: 03 abr. 2020.

CARVALHO, N. M.; NAKAGAWA, J. Sementes: ciência, tecnologia e produção. 5.ed. Jaboticabal: FUNEP, 2012. 590 p.

COMPANHIA NACIONAL DE ABASTECIMENTO - CONAB. Acompanhamento da safra brasileira de grãos - Safra 2019/20: quarto levantamento. Brasília, DF: Ministério da Agricultura, Pecuária e Abastecimento, 2020. v. 7, n. 4. 104 p.

DALOLIO, R. S.; BORIN, E.; CRUZ, R. M. S.; ALBERTON, O. Co-inoculação de soja com Bradyrhizobium e Azospirillum. Journal of Agronomic Sciences, Umuarama, v. 7, n. 2, p.17, 2018.

HUNGRIA, M.; CAMPO, R. J.; MENDES, I. C.; GRAHAM, P. H. Contribution of biological nitrogen fixation to the $\mathrm{N}$ nutrition of grain crops in the tropics: the success of soybean (Glycine $\max ($ L.) Merr.) in South America. In: SINGH, R. P.; SHANKAR, N.; JAIWAL, P. K. (ed.). Nitrogen nutrition and sustainable plant productivity. Houston: Studium, 2006. cap. 2, p. 43-93.

HUNGRIA, M.; NOGUEIRA, M. A.; ARAUJO, R. S. Co-inoculation of soybeans and common beans with rhizobia and azospirilla: strategies to improve sustainability. Biology and Fertility of Soils, Berlin, v. 49, n. 7, p.791-801, 2013. Disponível em: https://doi.org/10.1007/s00374-012-0771-5. Acesso em: 06 abr. 2020.

KOLCHINSKI, E. M., SCHUCH, L. O. B. Atributos de desempenho industrial e qualidade de sementes em aveia branca em função da disponibilização da adubação nitrogenada. Ciência 
Rural, Santa Maria, v. 33, n. 3, p.587-589, 2003. Disponível em: https://doi.org/10.1590/S0103-84782003000300031. Acesso em: 06 abr. 2020.

KOLCHINSKI E. M.; SCHUCH, L. O. B. Relações entre a adubação nitrogenada e a qualidade de grãos e de sementes em aveia branca. Ciência Rural, Santa Maria, v. 34, n. 2, p.979-383, 2004. Disponível em: https://doi.org/10.1590/S0103-84782004000200007. Acesso em: 06 abr. 2020.

KUMAR, R.; CHANDRA, R. Influence of PGPR and PSB on Rhizobium leguminosarum bv. viciae strain competition and symbiotic performance in lentil. World Journal of Agricultural Sciences, Aleppo, v. 4, n. 3, p.297-301, 2008.

MARCOS FILHO, J.; NOVEMBRE, A. D. L. C.; CHAMMA, H. M. C. P. Tamanho da semente e teste de envelhecimento acelerado para soja. Scientia Agricola, Piracicaba, v. 57, n. 3, p.473-482, 2000. Disponível em: https://doi.org/10.1590/S0103-90162000000300016. Acesso em: 06 abr. 2020.

MARCOS FILHO, J.; KIKUTI, A. L. P. Vigor de sementes de rabanete e o desempenho de plantas em campo. Revista Brasileira de Sementes, Londrina, v. 28, n. 3, p.44-51, 2006. Disponível em: https://doi.org/10.1590/S0101-31222006000300007. Acesso em: 06 abr. 2020.

MASCIARELLI, O.; URBANI, L.; REINOSO, H.; LUNA, V. Alternative mechanism for the evaluation of indole-3-acetic acid (IAA) production by Azospirillum brasilense strains and its effects on the germination and growth of maize seedlings. Journal of Microbiology, [s. l.], v. 51, n. 5, p.590-597, 2013. Disponível em: https://doi.org/10.1007/s12275-013-3136-3. Acesso em: 06 abr. 2020.

MATTUELlA, D.; SIMIONI, S. P.; SEGATTO, C.; CIGEL, C.; ADAMS, C. R.; KLEIN, C.; LAJÚS, C. R.; SORDI, A. Eficiência agronômica da cultura do trigo submetida a doses de nitrogênio em diferentes estádios ontogênicos. Ciência Agrícola, Alagoas, v. 16, n. 3, p.1-9, 2018.

NAKAGAWA, J. Testes de vigor baseados no desempenho das plântulas. In: KRZYZANOWSKI, F. C.; FRANÇA-NETO, J. B.; VIEIRA, D.V. (ed.). Vigor de sementes: conceitos e testes. Londrina: ABRATES, 1999. cap. 2, p. 2.1-2.24.

SÁ, M. E. Importância da adubação nitrogenada na qualidade de sementes. In: SÁ, M. E.; BUZZETTI, S. Importância da adubação na qualidade dos produtos agrícolas. São Paulo: Ícone, 1994. cap. 6, p. 65-98.

SANTOS NETO, J. T.; LUCAS, F. T.; FRAGA, D. F.; OLIVEIRA, L. F.; PEDROSO NETO, J. C. Adubação nitrogenada, com e sem inoculação de semente, na cultura da soja. FAZU em Revista, Uberaba, v. 2, n. 10, p.8-12, 2013.

SPONCHIADO, J. C.; SOUZA, C. A.; COELHO, C. M. M. Teste de condutividade elétrica para determinação do potencial fisiológico de sementes de aveia branca. Semina: Ciências Agrárias, Londrina, v. 35, n. 4, p.2405-2414, 2014. Disponível em: http://dx.doi.org/10.5433/1679-0359.2014v35n4Suplp2405. Acesso em: 07 abr. 2020. 
VIERA NETO, S. A.; PIRES, F. R.; MENEZES, C. C. E.; MENEZES, J. F. S.; SILVA, A. G.; SILVA, G. P.; ASSIS, R. L. Formas de aplicação de inoculante e seus efeitos sobre a nodulação da soja. Revista Brasileira de Ciência do Solo, Viçosa, v. 32, n. 2, p.861-870, 2008. Disponível em: https://doi.org/10.1590/S0100-06832008000200040. Acesso em: 06 abr. 2020.

ZAGO, L. F.; LIMA, C. R.; CRUZ, R. M. S.; ALBERTON, O. Inoculação de diferentes doses de Bradyrhizobium por cobertura e seu efeito na cultura da soja. Arquivos de Ciências Veterinárias e Zoologia da UNIPAR, Umuarama, v. 21, n. 2, p.65-69, 2018. Disponível em: https://doi.org/10.25110/arqvet.v21i2.2018.7302. Acesso em: 06 abr. 2020. 